\title{
Risk of Disease After Isoniazid Preventive Therapy for Mycobacterium tuberculosis Exposure in Young HIV-uninfected Children
}

\author{
Kany Kany A. Luabeya, MSc, * Michele D. Tameris, MB ChB, * Hennie D. Geldenhuys, M.Fam.Med, * \\ Humphrey Mulenga, MPH, * Amaryl Van Schalkwyk, * Elizabeth J. Hughes, * Asma Toefey, BA Hon., * \\ Thomas J. Scriba, PhD, * Gregory Hussey, PhD, * Hassan Mahomed, PhD, †t Helen McShane, PhD, $\S$ \\ Bernard Landry, MPH, $₫$ Willem A. Hanekom, FCP Paed, * and Mark Hatherill, MD*
}

\begin{abstract}
Background: The risk of developing tuberculosis (TB) disease in HIVuninfected children after isoniazid preventive therapy (IPT) for a positive QuantiFERON-TB Gold In-Tube test (QFT-GIT) is unknown. The aim of this study was to evaluate risk of TB disease after IPT in young HIV-uninfected children with a positive QFT-GIT result, or household TB contact.

Methods: HIV-uninfected South African infants aged 4-6 months were screened for enrolment in a TB vaccine trial. Baseline household TB contact and positive QFT-GIT result were exclusion criteria, and these infants were referred for IPT. Outcome data are reported for 36 months after IPT referral. Results: Four thousand seven hundred forty-nine infants were screened. Household TB contact was reported in $131(2.8 \%)$ infants; 279 (6.0\%) were QFT-GIT positive, and 138 of these 410 infants (34.0\%) started IPT. Forty-four cases of TB disease $(11.0 \%)$ were recorded within 991 child years of observation. TB disease incidence was 4.8 versus 3.6 per 100 child years in household exposed versus QFT-GIT-positive children [incidence rate ratio: 1.35; $95 \%$ confidence interval (CI): $0.67-2.88$ ] and 2.4 versus 5.5 per 100 child years in children who received versus did not receive IPT, respectively (incidence rate ratio: 0.44; 95\% CI: 0.17-0.96). Adjusted hazard ratio (Cox regression) for TB disease was 0.48 (95\% CI: $0.21-1.05)$ for those who received IPT.

Conclusion: In young HIV-uninfected children, the effect of IPT on risk of TB disease is similar, whether TB exposure was defined by household contact history or by positive QFT-GIT result. International IPT guidelines for HIV-uninfected children with a positive QFT-GIT result should be updated.
\end{abstract}

Key Words: IPT, Quantiferon-TB Gold In Tube, children, tuberculosis, Mycobacterium tuberculosis

(Pediatr Infect Dis J 2015;34:1218-1222)

$T^{4}$ uberculosis (TB) is a major cause of morbidity and mortality in developing countries, with 1.3 million deaths reported in 2012 (WHO Report 2013). In TB endemic regions, despite Calmette-Guérin bacillus (BCG) vaccination, the risk of pulmonary TB disease in young children remains high after exposure to Mycobacterium tuberculosis (Mtb). ${ }^{1}$ The natural history of TB, described from observational studies before the chemoprophylaxis era, shows that young age is an important determinant of risk for TB disease in immune competent children. ${ }^{2}$ The youngest children are also at greatest risk of severe morbidity, including miliary TB and meningitis. ${ }^{3,4}$

Accepted for publication February 16, 2015 .

From the *South African Tuberculosis Vaccine Initiative (SATVI), Institute of Infectious Disease and Molecular Medicine (IDM), University of Cape Town; †Western Cape Government; †Stellenbosch University, Cape Town, South Africa; §Jenner Institute, Nuffield Department of Clinical Medicine, University of Oxford, Oxford, United Kingdom; and $\uparrow$ Aeras, Rockville, Maryland.

The authors have no funding or conflicts of interest to disclose.

Address for correspondence: Kany Kany A. Luabeya, MSc, South African Tuberculosis Vaccine Initiative, Brewelskloof Hospital, Haarlem Street, Worcester 6850, South Africa. E-mail: angelique.luabeya@uct.ac.za.

Copyright (C) 2015 Wolters Kluwer Health, Inc. All rights reserved.

ISSN: $0891-3668 / 15 / 3411-1218$

DOI: $10.1097 / \mathrm{INF} .0000000000000874$
Isoniazid preventive therapy (IPT) is thought to reduce the risk of TB disease in children who become infected after Mtb exposure. ${ }^{5}$ In a systematic review by Smieja et al, IPT provided $90 \%$ protection against TB disease in children given prophylaxis. ${ }^{67}$ A similar effect was described in HIV-infected children. ${ }^{8,9}$ Efficacy trials of TB preventive therapy have also shown that IPT reduces the risk of TB disease in HIV-uninfected individuals by $60 \%$ over 2 years of follow-up. ${ }^{7}$ In TB hyperendemic settings, children may be exposed and infected with $M t b$ from infancy. ${ }^{10}$ Therefore, the World Health Organization (WHO) recommends that every child younger than 5 years who is exposed to an adult TB case should be given chemoprophylaxis in the form of IPT after exclusion of active TB disease. ${ }^{25}$ South African national guidelines recommend IPT for HIV-uninfected children younger than age 5 years who have $M t b$ exposure, defined by a history of close contact with a person with active TB disease or a positive Mantoux tuberculin skin test (TST). ${ }^{11}$ A positive QuantiFERON-TB Gold In-Tube Test (QFT-GIT, Cellestis Limited, Chadstone, Australia) result is not currently included in national guidelines as an indication for IPT for HIV-uninfected children. IPT is not recommended for $M t b$-exposed, HIV-uninfected adults in South Africa and many other high TB burden countries, because of the likelihood of repeated re-exposure to $M t b$.

Interferon-gamma release assays (IGRAs), such as the QFTGIT, have sometimes been used for confirmation of $M t b$ exposure and diagnosis of $M t b$ infection in low-burden countries and more commonly, in research settings, in high-burden countries. ${ }^{12,13}$ The WHO has recently recommended that IGRAs should not replace the TST in low-income and middle-income countries for the diagnosis of latent TB infection in childre ${ }^{25}$ However, there is a lack of data on the effect of IPT on risk of TB disease in young children with a positive QFT-GIT result in developing countries with a high TB prevalence. These data are crucial to guide programmatic decisions on whether young children with a positive QFT-GIT result should also receive IPT. This study evaluates the risk of TB disease after IPT in young, HIV-uninfected South African children with $M t b$ exposure, as documented by household contact with an adult TB patient, or by a positive QFT-GIT result.

\section{METHODS}

This analysis was conducted on infants screened for participation in a phase IIb double-blind randomized, controlled clinical trial of a novel TB vaccine (MVA85A), which has been described previously. ${ }^{14}$ The study was conducted in a rural region of the Western Cape, South Africa, where the annual TB incidence is nearly $1 \%$ in adults (993 per 100,000 in 2012) ${ }^{14}$ and approximately $3 \%$ among children younger than 5 years of age. ${ }^{15}$ The study was approved by the Human Research Ethics Committee of the University of Cape Town. ${ }^{14}$ Written informed consent was received from parents for access to medical records. Infants were 126-182 days old at the time of screening, with a weight-for-age $>3$ rd percentile, 
generally good health and had completed the age-appropriate childhood immunization schedule recommended by the South African Department of Health, including BCG vaccination within 7 days of birth. Infants with acute or chronic illness, HIV infection confirmed by HIV polymerase chain reaction and history or evidence of active TB disease were excluded.

All infants with known $M t b$ exposure, defined by a history of close household TB contact or a positive QFT-GIT result, who form the basis of this analysis, were excluded from the vaccine trial and were referred for IPT. Infants with a history of close household TB contact did not undergo an additional QFT-GIT or TST, as the criteria for exclusion was already met. Thus, the proportion of infants with a history of household TB contact who tested QFT-GIT positive cannot be calculated. Infants without a history of household contact or other exclusion criteria underwent an additional QFT-GIT. Thereafter, all infants with $M t b$ exposure, whether documented by history of household contact or positive QFT-GIT, were given a referral letter to notify the local clinic, recommending that IPT should be provided. At the clinic level, children were further investigated by TST and chest radiograph (to exclude active disease) before provision of IPT. After completion of the trial, outcome data on all infants referred for IPT, including date of starting IPT and any treatment for subsequent diagnosis of TB disease, were collected from the TB notification and treatment registers for 36 months after screening. TB diagnosis was made on clinical, radiological and microbiological grounds, based on the judgment of the treating physician.

IPT and TB disease treatment status were analyzed as categorical variables, and proportions were compared between infants with $M t b$ exposure, defined by either positive QFT-GIT or history of household TB contact (HHC), using the $\chi^{2}$ test in the univariate analysis. A Student's $t$ test was used for normally distributed continuous variables and the Kruskal-Wallis test for non-normally distributed continuous variables. Cox regression analysis was used to estimate the risk of TB disease through 36 months after referral for IPT. For the purpose of this analysis, the sample mean was imputed for missing continuous variables $(n=49)$ and a 0 value (nonexposed) for missing categorical data. Data were analyzed using Stata version 11.2 (StataCorp LP, StataCorp. 2009, Stata Statistical Software: Release 11, College Station, TX).

\section{RESULTS}

Four thousand seven hundred forty-nine infants were screened. The 410 infants $(8.6 \%)$ with either a positive QFTGIT result or history of $\mathrm{HHC}$ at baseline are included in this analysis. The mean (standard deviation) age at screening was
130 (32.5) days; $46 \%(\mathrm{n}=187)$ of infants were male; $80 \%$ $(n=326)$ had mixed race ancestry; and 20\% $(n=84)$ had black African ancestry. Mean (standard deviation) weight at screening was $6.3(0.97) \mathrm{kg}$.

A HHC was reported in $131(2.8 \%)$ infants. An additional 279 infants were QFT-GIT positive $(6.0 \%)$. Despite referral for IPT, only one third $(n=138)$ of the 410 infants had receipt of IPT documented at the clinic. The median time from screening to start IPT was 118 [interquartile range (IQR): 77-178] days. A significantly higher proportion of infants with $\mathrm{HHC}(50 \% ; n=65)$ were documented to have received IPT, compared with $26 \%(\mathrm{n}=73)$ of QFT-GIT-positive infants $(P<0.001)$.

Forty-four incident cases of TB disease (11.0\%) were recorded within 991 child years of observation, after screening and referral for IPT, yielding an overall annual incidence rate of 4.4 (95\% CI: 3.0-6.0) per 100 child years. The median (IQR) age at TB diagnosis was 201 (158-404) days. Among children who received IPT, the only difference in baseline characteristics by TB disease outcome was the mean weight-for-age $\mathrm{Z}$ score, with children who developed TB having the lowest weight-forage $\mathrm{Z}$ score (Table 1). However, children who subsequently developed TB disease had longer median time from screening to IPT uptake than those who did not develop TB disease [262 (84-447) vs. 117 (69-173) days], but this was not statistically significant $(P=0.1)$.

The distribution of TB cases by baseline TB exposure criteria (HHC or QFT-GIT positive), and by receipt of IPT, is shown in Figure 1.

A summary of additional TB investigations performed at clinic level is shown in Figure 2. Among children who received IPT (investigation data available for $\mathrm{n}=137), 118(86 \%)$ had a TST and $36(26 \%)$ had a chest radiograph; although among children who did not receive IPT (investigation data available for $n=272$ ), only 148 (54\%) had a TST and $59(21.6 \%)$ a chest radiograph. A high proportion of children with a positive QFT-GIT result also had a TST $(\mathrm{n}=112,64 \%)$ but did not receive IPT.

Twelve cases (12 of $44 ; 27.3 \%$ ) of TB disease occurred in children with a HHC, compared with 32 of $44(72.7 \%)$ cases in children with a positive QFT-GIT result. The annual TB disease incidence rate was not significantly different between children with $M t b$ exposure defined by HHC [3.6 (95\% CI: 2.0-6.3) per 100 child years] and those with a positive QFT-GIT result [4.8 (95\% CI: 3.4$6.0)$ per 100 child years], with an incidence rate ratio of $1.35(95 \%$ CI: 0.68-2.88). The median (IQR) age at TB diagnosis was not significantly different between children with a HHC [median 231 (130-561) days] and children with a positive QFT-GIT [median 196 (164-377) days] $(P=0.9)$.

TABLE 1. Baseline Characteristics at Screening of Children Who Received IPT, by TB Disease Outcome $\left(\mathrm{n}=137^{*}\right)$

\begin{tabular}{lccc}
\hline \hline Variables & $\begin{array}{c}\text { TB Disease } \\
(\mathrm{n}=8)\end{array}$ & $\begin{array}{c}\text { No. TB Disease } \\
(\mathrm{n}=129)\end{array}$ & $P$ Value \\
\hline Age at baseline (mo), median (IQR) & $135(129-153)$ & $129(127-135)$ & 0.09 \\
Gender, male & $4(50)$ & $57(44)$ & 0.7 \\
Weight-for-age Z score at baseline, mean (SD) & $-2.67(0.65)$ & $0.03(0.98)$ & 0.02 \\
History of household contact & $4(50)$ & $61(47)$ & 0.8 \\
QFT-GIT positive (n = 279) & $4(50)$ & $68(53)$ & 0.6 \\
Black African & $2(25)$ & $24(19)$ & \\
Mixed race & $6(75)$ & $105(81)$ & 0.1 \\
Time to IPT initiation (d), median (IQR) & $262(84-447)$ & $111(86)$ & 0.9 \\
TST record available at clinic & $7(88)$ & $32(25)$ & 0.1 \\
CXR record available at clinic & $4(50)$ & & \\
\hline
\end{tabular}

Categorical data are shown as $\mathrm{n}(\%)$

SD indicates standard deviation; CXR, chest radiograph. 

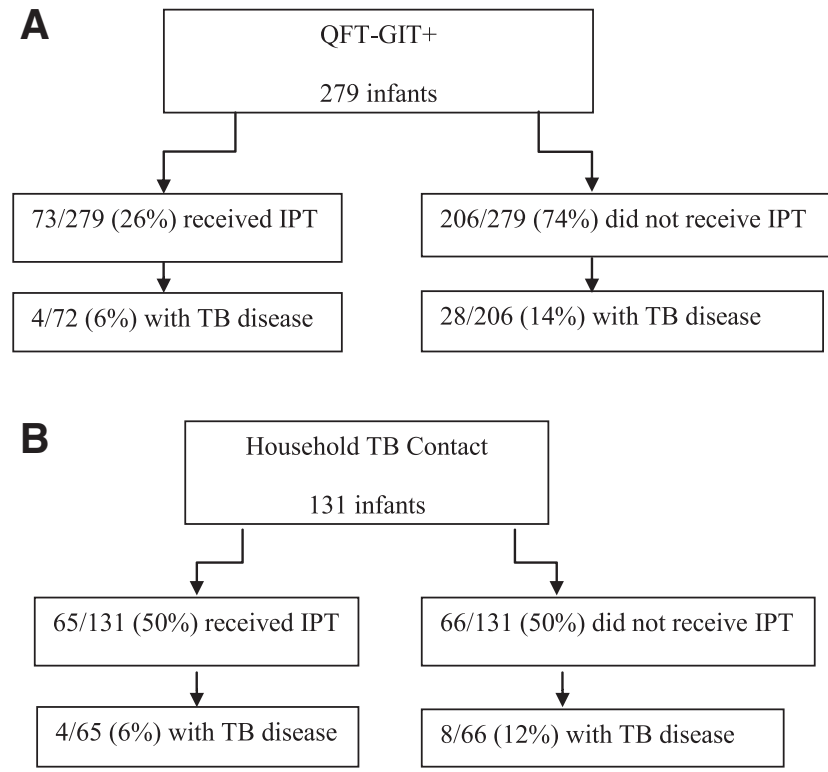

FIGURE 1. IPT and TB disease status in infants with a positive QFT-GIT and a history of household TB exposure.

Eight cases ( 8 of $44 ; 18.2 \%$ ) of TB disease occurred in children who received IPT, compared with 36 of $44(81.8 \%)$ cases in children who did not receive IPT. The annual TB disease incidence rate was 2.4 (95\% CI: 1.2-4.8) per 100 child years in children who received IPT, compared with 5.4 (95\% CI: 3.9-7.5) per 100 child years in children who did not receive IPT [incidence rate ratio: 0.44 (95\% CI: 0.17-0.96)]. The median (IQR) age at TB diagnosis was not significantly different between children who received IPT [median 172 (IQR: 149-255) days] and children who did not receive IPT [median 201 (IQR: 61-404) days] $(P=0.2)$. Among those children who started IPT, 8 cases of TB disease occurred within 6 months of starting IPT [incidence rate: 13 (95\% CI: 6-25) per 100 child years observation], and no cases of TB disease were recorded thereafter.

In the univariate analysis, both IPT and older age were associated with a statistically significant reduction in the risk of TB disease (Table 2). In the adjusted Cox regression model, age remained significantly associated with independent hazard for TB disease, with a $47 \%$ reduction in risk per additional month of age at baseline (Table 3). Although the adjusted hazard of TB disease was also reduced by more than $50 \%$ in children receiving IPT, compared with children who did not receive IPT, this difference was not statistically significant $(P=0.06)$

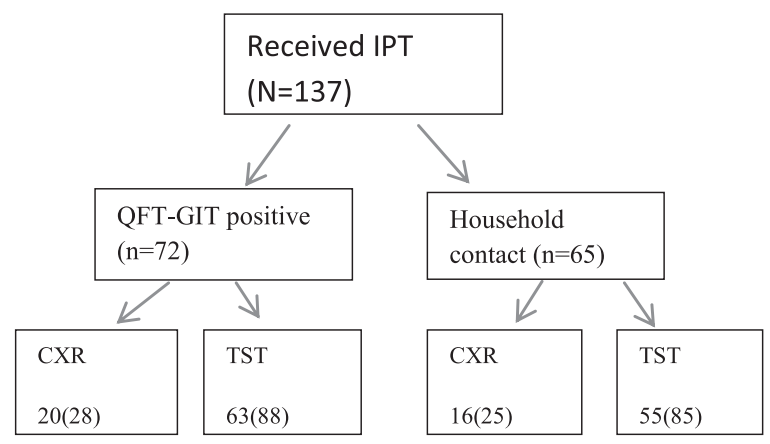

TABLE 2. Univariate Analysis of Factors Associated with Risk of TB Disease

\begin{tabular}{lccc}
\hline \hline & $\begin{array}{c}\text { Unadjusted } \\
\text { HR }\end{array}$ & $95 \%$ CI & P Value \\
& 0.58 & $0.39-0.87$ & $\mathbf{0 . 0 0 9}$ \\
Age at baseline (mo) & 0.70 & $0.40-1.34$ & 0.30 \\
Gender, male & 0.70 & $0.5-1.01$ & 0.05 \\
$\quad$ Weight-for-age Z score (kg) & & & \\
$\quad$ at baseline & & & \\
Exposure to TB & 1.00 & & \\
$\quad$ History of household contact & 1.70 & $0.86-3.28$ & 0.10 \\
$\quad$ QFT-GIT positive (n=279) & 0.45 & $0.20-0.97$ & $\mathbf{0 . 0 4}$ \\
IPT (yes) & & & \\
Ethnicity & 1.00 & & \\
$\quad$ Black African & 0.72 & $0.36-1.42$ & 0.30 \\
$\quad$ Mixed race &
\end{tabular}

Bold values indicate statistical significance at $P<0.05$.

HR indicates hazard ratio.

TABLE 3. Multivariate Analysis of Factors Associated With Risk of TB Disease, Cox Regression Model

\begin{tabular}{lccl}
\hline \hline & $\begin{array}{c}\text { Adjusted } \\
\text { HR }\end{array}$ & 95\% CI & P Value \\
& 0.53 & $0.34-0.81$ & $\mathbf{0 . 0 0 4}$ \\
Age at baseline (mo) & 0.92 & $0.49-1.75$ & 0.80 \\
Gender, male & 0.70 & $0.49-1.01$ & 0.057 \\
$\quad$ Weight Z score (kg) at baseline & & & \\
Exposure to TB & 1.00 & & \\
$\quad$ History of household contact & 1.67 & $0.84-3.30$ & 0.10 \\
$\quad$ QFT-GIT positive (n = 279) & 0.48 & $0.21-1.05$ & 0.06 \\
IPT (yes) & & & \\
Ethnicity & 1.00 & & \\
$\quad$ Black & 0.68 & $0.33-1.40$ & 0.30 \\
$\quad$ Mixed race &
\end{tabular}

Bold value indicates statistical significance at $P<0.05$.

HR indicates hazard ratio.

\section{DISCUSSION}

We have shown that in this TB hyperendemic setting, more than $8 \%$ of healthy, BCG-vaccinated, HIV-uninfected South African infants have evidence of $M t b$ exposure before 6 months of age, including $6 \%$ who were shown to have evidence of recent TB infection on the basis of a positive QFT-GIT result. Given the evidence of very high $M t b$ transmission rates in this population, it is not surprising that the incidence of TB disease in these young children has been shown to exceed $4 \%$ per annum. ${ }^{14,15}$ Despite written referral for IPT, only one third of children who were referred for IPT had documentation of receiving IPT. In particular, very few children referred for a positive QFT-GIT result received IPT, despite having

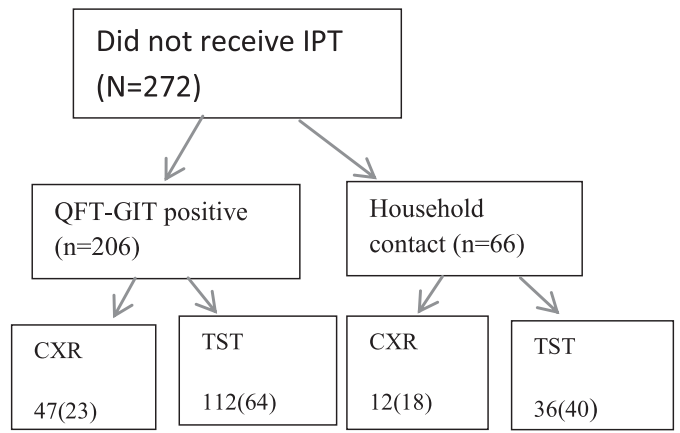

FIGURE 2. Additional clinic investigations, including TST and chest radiograph (CXR), by IPT status $(n=409)$. 
an additional TST performed at the clinic, although the nonprovision of IPT to these children might be explained by discordance between QFT-GIT and TST results. ${ }^{16}$ Furthermore, because a positive QFT-GIT result is not currently included in the South African national guidelines for IPT administration to HIV-uninfected, $M t b$ exposed children younger than 5 years (SA National Tuberculosis Guidelines 2009), it is likely that lack of familiarity with this test result at the local clinic level contributed to the lower rate of IPT administration. However, even among those children with a known history of household contact, only 50\% received IPT, implying that other factors are also at play. Programmatic measures to improve provision and adherence of chemoprophylaxis for $M t b$-exposed children are needed ${ }^{17}$

Children who received IPT had more than 50\% reduction in hazard for TB disease, compared with those who did not receive IPT. However, after adjusting for potential confounders, the effect of IPT on hazard for TB disease was similar, irrespective of whether the exposure was defined by a history of household TB contact or by positive QFT-GIT result. It is notable that among children who received IPT, all cases of TB disease were diagnosed within 6 months of starting IPT, implying that these were prevalent rather than incident cases that might not be prevented by isoniazid monotherapy. This observation is supported by the finding that children who were diagnosed with TB after IPT referral were relatively undernourished at baseline, which is consistent with active TB disease. However, these results are in contrast to those reported in a mass IPT trial among adult miners in South Africa, in which TB incidence increased shortly after completion of IPT. ${ }^{18}$

As fewer QFT-GIT-positive children actually received IPT after referral, compared with children with a history of household contact, we postulate that the reduction in risk of TB disease might have been even greater in QFT-GIT-positive children if health service IPT prescribing patterns had been equal. At the very least, because hazard of TB disease was similar after adjusting for receipt of IPT, children with a positive QFT-GIT result would be expected to receive similar benefit from administration of IPT as children with a history of household TB contact. Therefore, despite the recent WHO recommendation that IGRAs should not replace the TST for diagnosis of latent TB infection in children in low-income and middle-income countries, ${ }^{25}$ our findings support further research into the use of QFT-GIT results in programmatic algorithms to guide IPT provision to $M t b$-exposed, HIV-uninfected children - particularly those younger than 5 years of age.

The very high rate of baseline positive QFT-GIT result (6\%) in otherwise healthy infants younger than 6 months of age is surprising, especially considering that infants already identified as $M t b$ exposed by history of household TB contact were excluded from QFT-GIT. Given the reported variability in sensitivity of the QFTGIT in infants, one explanation for this high prevalence might be a high number of false-positive QFT-GIT results. ${ }^{19}$ However, this possibility is not supported by the high incidence of subsequent TB disease and observed beneficial effects of IPT. $M t b$ infection is known to be common in young children in hyperendemic TB settings in the absence of a documented source case. ${ }^{20,21}$

This study has certain limitations, which should be considered when interpreting these findings. The Western Cape of South Africa is a very high TB incidence area that may represent the extreme of TB transmission, and therefore, our findings may not be applicable to lower TB burden countries. Although baseline measures to exclude TB disease and define $M t b$ exposure were robust, children with HHC did not undergo a QFT-GIT. Therefore, it is not possible to examine the relationship between exposure defined by HHC and that defined by QFT-GIT. Second, because children who were identified as $M t b$ exposed at baseline did not undergo further follow-up visits, outcome data were collected retrospectively at the end of the clinical trial. Therefore, it is possible that some cases of TB were missed and also that some children diagnosed and treated for TB on clinical grounds might not have been classified as true TB cases by a study algorithm in a research setting. Detailed data on IPT adherence and completion rates are lacking. Children with no record of IPT at the clinic were considered as not having received IPT, although they may have moved out of the study area and received IPT elsewhere. It is likely that improved IPT adherence rates, whether in the context of study-specific recommendations or programmatic health service interventions, might have accentuated the effects of IPT on reduction in risk of TB disease. It must be acknowledged that there are few validated studies to guide interpretation of the QFT-GIT in pediatric populations, particularly in respect of the $0.35 \mathrm{IU} / \mathrm{mL}$ threshold for positivity. ${ }^{12}$ However, we note that it is the sensitivity, not specificity, of IGRAs that are thought be lower in high TB burden countries, compared with low-burden countries. ${ }^{22}$

Because IGRAs are used in developing countries, particularly in research settings, ${ }^{23}$ there is a need for additional evidence to define the role of QFT-GIT results in programmatic algorithms that guide IPT provision for Mtb-exposed, HIV-uninfected children. However, given the scientific plausibility of benefit from IPT, the supporting data from clinical trials in HIV-infected persons $s^{9,11,24}$ and uncontrolled studies, such as that reported here, a controlled trial of IPT, would be ethically problematic in this pediatric study population. We do not propose that the QFT-GIT should replace the TST for diagnostic purposes in low-income and middle-income countries. However, we do suggest that HIV-uninfected children younger than 5 years of age who have a positive QFT-GIT result should be considered for IPT, regardless of TB contact history and TST result.

\section{ACKNOWLEDGMENT}

Authors'contribution: A.L. analyzed the data. A.L. and M.H. wrote the first draft manuscript. All authors planned and implemented the study and contributed to the final manuscript.

\section{REFERENCES}

1. Marais BJ, Graham SM, Maeurer M, et al. Progress and challenges in childhood tuberculosis. Lancet Infect Dis. 2013;13:287-289.

2. Marais BJ, Gie RP, Schaaf HS, et al. The natural history of childhood intra-thoracic tuberculosis: a critical review of literature from the prechemotherapy era. Int J Tuberc Lung Dis. 2004;8:392-402.

3. Cruz AT, Starke JR. Clinical manifestations of tuberculosis in children. Paediatr Respir Rev. 2007;8:107-117.

4. Moyo S, Verver S, Mahomed H, et al. Age-related tuberculosis incidence and severity in children under 5 years of age in Cape Town, South Africa. Int J Tuberc Lung Dis. 2010;14:149-154

5. Ayieko J, Abuogi L, Simchowitz B, et al. Efficacy of isoniazid prophylactic therapy in prevention of tuberculosis in children: a meta-analysis. $B M C$ Infect Dis. 2014;14:91

6. Hill PC, Rutherford ME, Audas R, et al. Closing the policy-practice gap in the management of child contacts of tuberculosis cases in developing countries. PLoS Med. 2011;8:e1001105.

7. Smieja MJ, Marchetti CA, Cook DJ, et al. Isoniazid for preventing tuberculosis in non-HIV infected persons. Cochrane Database Syst Rev. 2000 CD001363.

8. Zar HJ, Cotton MF, Strauss S, et al. Effect of isoniazid prophylaxis on mortality and incidence of tuberculosis in children with HIV: randomised controlled trial. BMJ. 2007;334:136.

9. Gray DM, Zar H, Cotton M. Impact of tuberculosis preventive therapy on tuberculosis and mortality in HIV-infected children. Cochrane Database Syst Rev. 2009: CD006418.

10. Perez-Velez CM, Marais BJ. Tuberculosis in children. $N$ Engl J Med 2012;367:348-361.

11. Schaaf HS, Cotton MF, Boon GP, et al. Isoniazid preventive therapy in HIVinfected and -uninfected children (0-14 years). S Afr Med J. 2013;103:714-715. 
12. Mahomed H, Hawkridge T, Verver S, et al. The tuberculin skin test versus QuantiFERON TB Gold $\AA$ in predicting tuberculosis disease in an adolescent cohort study in South Africa. PLoS One. 2011;6:e17984.

13. Starke JR. Interferon- $\gamma$ release assays for the diagnosis of tuberculosis infection in children. J Pediatr. 2012;161:581-582.

14. Tameris MD, Hatherill M, Landry BS, et al; MVA85A 020 Trial Study Team. Safety and efficacy of MVA85A, a new tuberculosis vaccine, in infants previously vaccinated with BCG: a randomised, placebo-controlled phase 2 b trial. Lancet. 2013;381:1021-1028.

15. Hawkridge A, Hatherill M, Little F, et al; South African BCG trial team. Efficacy of percutaneous versus intradermal BCG in the prevention of tuberculosis in South African infants: randomised trial. BMJ. 2008;337:a2052.

16. Sollai S, Galli L, de Martino M, et al. Systematic review and meta-analysis on the utility of Interferon-gamma release assays for the diagnosis of Mycobacterium tuberculosis infection in children: a 2013 update. BMC Infect Dis 2014;14 (Suppl 1):S6.

17. van Soelen N, du Preez K, van Wyk SS, et al. Does an isoniazid prophylaxis register improve tuberculosis contact management in South African children? PLoS One. 2013;8:e80803.

18. Churchyard GJ, Fielding KL, Lewis JJ, et al; Thibela TB Study Team. A trial of mass isoniazid preventive therapy for tuberculosis control. N Engl J Med. 2014;370:301-310.
19. Bergamini BM, Losi M, Vaienti F, et al. Performance of commercial blood tests for the diagnosis of latent tuberculosis infection in children and adolescents. Pediatrics. 2009; 123:e419-e424.

20. Hesseling AC, Mandalakas AM, Kirchner HL, et al. Highly discordant T cell responses in individuals with recent exposure to household tuberculosis. Thorax. 2009;64:840-846.

21. Marais BJ, Hesseling AC, Schaaf HS, et al. Mycobacterium tuberculosis transmission is not related to household genotype in a setting of high endemicity. J Clin Microbiol. 2009;47:1338-1343.

22. Machingaidze S, Wiysonge CS, Gonzalez-Angulo Y, et al. The utility of an interferon gamma release assay for diagnosis of latent tuberculosis infection and disease in children: a systematic review and meta-analysis. Pediatr Infect Dis J. 2011;30:694-700.

23. Mahomed H, Ehrlich R, Hawkridge T, et al. TB incidence in an adolescent cohort in South Africa. PLoS One. 2013;8:e59652.

24. van Halsema CL, Fielding KL, Chihota VN, et al. Tuberculosis outcomes and drug susceptibility in individuals exposed to isoniazid preventive therapy in a high HIV prevalence setting. AIDS. 2010;24:1051-1055.

25. Guidance for National Tuberculosis Programmes on the Management of Tuberculosis in Children, 2nd ed. World Health Organization; 2014. Available at: http://apps.who.int/iris/bitstream/10665/112360/1/9789241548748_eng. pdf?ua=1. Accessed November 24, 2014.

\section{Current Abstracts}

\section{Edited by: Robert J. Leggiadro, $M D$}

\section{Outbreaks of Diseases Associated with Recreational Water- US, 2011-2012}

Centers for Disease Control and Prevention. MMWR. 2015;64:668-672.

Outbreaks of diseases associated with recreational water use result from exposure to chemicals or infectious pathogens in recreational water venues that are treated (eg, pools and hot tubs or spas) or untreated (eg, lakes and oceans). For 2011-2012, the most recent years for which finalized data are available, public health officials from 32 states and Puerto Rico reported 90 recreational waterassociated outbreaks to Centers for Disease Control and Prevention's (CDC) Waterborne Disease and Outbreak Surveillance System via the National Outbreak Reporting System (NORS). A recreational water-associated outbreak is the occurrence of similar illnesses in 2 or more persons, epidemiologically linked by location and time of exposure to recreational water or recreational water-associated chemicals volatilized into the air surrounding the water. In 2010 , waterborne outbreaks became nationally notifiable.

The 90 outbreaks resulted in at least 1788 cases, 95 (5\%) hospitalizations and 1 death. Etiology was confirmed for 73 (81\%) outbreaks: 69 (77\%) outbreaks were caused by infectious pathogens, including 2 outbreaks with multiple etiologies, and 4 (4\%) by chemicals. Among the outbreaks caused by infectious pathogens, 37 (54\%) were caused by Cryptosporidium.

Outbreaks associated with treated recreational water accounted for 69 (77\%) of the 90 outbreaks reported for 2011-2012 and resulted in at least 1309 cases, 73 hospitalizations and 1 death. The median number of cases reported for these outbreaks was 7 (2-144 cases). Hotels (eg, hotel, motel, lodge or inn) were the setting of $13(19 \%)$ of the treated recreational water-associated outbreaks. Twelve $(92 \%)$ of these outbreaks started outside of June-August; $10(77 \%)$ were at least in part associated with a spa. Among the 69 outbreaks, 36 (52\%) were caused by Cryptosporidium. The 69 outbreaks had a seasonal distribution, with $42(61 \%)$ starting in
June-August. Acute gastrointestinal illness was the disease manifestation in $34(81 \%)$ of these summer outbreaks, with Cryptosporidium causing 32 (94\%) of them.

For 2011-2012, 21 (23\%) outbreaks were associated with untreated recreational water. These outbreaks resulted in at least 479 cases and 22 hospitalizations. The median number of cases reported for these outbreaks was 16 (range: $2-125)$. Twenty $(95 \%)$ of these outbreaks were associated with fresh water, 18 (86\%) began in June-August and 7 (33\%) were caused by Escherichia coli $0157:$ H7 or 0111.

Comment: In the US, codes regulating public, treated recreational water venues are independently written and enforced by individual state or local agencies. Consequent variation in codes is a potential barrier to preventing or controlling outbreaks associated with these venues. In August 2014, CDC released the first edition of the Model Aquatic Health Code (http:// www.cdc.gov/mahc), a comprehensive set of science-based and best-practice recommendations to reduce risk for illness and injury at public, treated recreational water venues.

Among treated recreational water-associated outbreaks of gastrointestinal illness that began in June-August, $>90 \%$ were caused by Cryptosporidium, an extremely chlorine-tolerant parasite that can survive at CDC-recommended chlorine levels $(1-3 \mathrm{mg} / \mathrm{L})$ and $\mathrm{pH}(7.2-7.8)$ for $>10$ days. Systematic national genotyping and subtyping of Cryptosporidium in clinical specimens and environmental samples through CryptoNet (http:// www.cdc.gov/parasites/crypto/cryptonet.html) can identify circulating Cryptosporidium species and subtypes and help identify epidemiologic linkages between reported cases. Molecular typing could substantially help elucidate cryptosporidiosis epidemiology in the US and inform development of future guidance to prevent recreational water-associated and other outbreaks of cryptosporidiosis. 